\title{
Improved BBN constraints on the variation of the gravitational constant
}

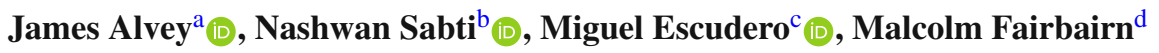 \\ Theoretical Particle Physics and Cosmology Group, Department of Physics, King's College London, Strand, London WC2R 2LS, UK
}

Received: 19 December 2019 / Accepted: 6 February 2020 / Published online: 19 February 2020

(C) The Author(s) 2020

\begin{abstract}
Big Bang Nucleosynthesis (BBN) is very sensitive to the cosmological expansion rate. If the gravitational constant $G$ took a different value during the nucleosynthesis epoch than today, the primordial abundances of light elements would be affected. In this work, we improve the bounds on this variation using recent determinations of the primordial element abundances, updated nuclear and weak reaction rates and observations of the Cosmic Microwave Background (CMB). When combining the measured abundances and the baryon density from CMB observations by Planck, we find $G_{\mathrm{BBN}} / G_{0}=0.99_{-0.05}^{+0.06}$ at $2 \sigma$ confidence level. If the variation of $G$ is linear in time, we find $\dot{G} / G_{0}=$ $0.7_{-4.3}^{+3.8} \times 10^{-12}$ year $^{-1}$, again at $2 \sigma$. These bounds are significantly stronger than those from previous primordial nucleosynthesis studies, and are comparable and complementary to $\mathrm{CMB}$, stellar, solar system, lunar laser ranging, pulsar timing and gravitational wave constraints.
\end{abstract}

\section{Introduction}

Ever since the Large Number Hypothesis of Dirac, physicists have wondered whether the constants of nature may evolve over time [1]. Since then the mathematical framework for such a variation has been developed. This was done first with ideas such as Kaluza-Klein theory where the coupling between gravity and matter sectors was set by the size of a compact dimension [2,3], and then by advancements such as Brans-Dicke theory where the coupling between matter and gravity is endowed with dynamics [4]. A key problem in the ongoing attempt to unify the four forces of nature using string theory is figuring out how to stabilise the higher

\footnotetext{
a e-mail: james.alvey@kcl.ac.uk

be-mail: nashwan.sabti@kcl.ac.uk (corresponding author)

c e-mail: miguel.escudero@kcl.ac.uk

de-mail: malcolm.fairbairn@kcl.ac.uk
}

dimensions, and consequently the value of the low energy couplings, including the gravitational constant $G$ [5].

A theory where $G$ is allowed to vary almost always involves (by definition) promoting $G$ to be related to the expectation value of some dynamical scalar field. For consistency, the scalar field will then have a kinetic term and a potential. In order to observe any dynamical changes of such a field over cosmological time, the curvature of that potential, and therefore the mass of this field, has to be very small. Constraints from solar system observations, like radar ranging of Mars, place very tight limits on the contribution to gravity due to such light scalars in the Universe today [6]. ${ }^{1}$

On the other hand, we are in possession of much less information about the detailed behaviour of gravity in the early Universe, in particular during the period of Big Bang Nucleosynthesis at which point the Universe is expected to be extremely uniform. Most scenarios where the value of $G$ is set by a scalar field obtaining an expectation value result in an effective mass for that field that is much larger than the energy scales relevant even at this earlier epoch. As such, we do not have any good reason to expect that $G$ is likely to be different. Nevertheless, if we have the technology to place new limits on the variation of a fundamental constant like this one, we should take the opportunity to obtain those limits to the best of our abilities. The subject may also become more important in the coming years given upcoming atomic interferometry experiments which will test extensions of gravity [8].

Strictly speaking, since $G$ is dimensionful, we should be very careful when we discuss its time variation. This is because any physically meaningful change in couplings should be written as a change in the dimensionless ratio between two dimensionful quantities. We are assuming a scenario where $G=M_{\mathrm{Pl}}^{-2}$ changes while none of the particle physics energy scales vary. For example, consider the

\footnotetext{
${ }^{1}$ Notable exceptions to this are Chameleon type theories where the mass of such scalar fields can be affected by the local density of matter [7].
} 
case where all the dimensionful parameters of the Standard Model (like the Brout-Englert-Higgs expectation value $v$ and $\Lambda_{\mathrm{QCD}}$ ) are determined by dimensional transmutation of couplings set at a single energy scale $M_{\mathrm{GUT}}$. If gravitational corrections to the running of those couplings are irrelevant, then discussing variations in $M_{\mathrm{Pl}} / M_{\mathrm{GUT}}$ becomes better defined [9]. This is the kind of situation we are considering. In what follows, we will refer to $G$ as the value of Newton's gravitational constant over time and $G_{0}$ as its value as measured today.

Big Bang Nucleosynthesis (BBN) is sensitive to modifications of the expansion history in the early Universe. Indeed, predictions for the primordial element abundances are strongly dependent on the Hubble rate $H$ and subsequently on the value of the gravitational constant $G$ during the relevant epoch. Recent improvements in measurements of these early Universe abundances [10-16] allow us to improve constraints on the gravitational physics during the BBN era. With some mild assumptions detailed in the Sect. 3, this can be translated into bounds on the variation in the value of $G$ between nucleosynthesis and today.

Nucleosynthesis is of course not the only scenario in which changes in $G$ would be manifest. Bounds on the time variation of $G$ can be inferred from Cosmic Microwave Background (CMB) [17,18], stellar [19-21], solar system [22-24], pulsar timing [25,26], lunar laser ranging [27,28] and gravitational wave $[29,30]$ measurements. These place constraints at the same order of magnitude as those from BBN [31-34], with the strongest bound being $\dot{G} / G_{0}=$ $(7.1 \pm 7.6) \times 10^{-14}$ year $^{-1}$ at the $68 \%$ CL from lunar laser ranging experiments [28]. Note that each given constraint probes different epochs in the evolution of the Universe, and should be compared as such. For comprehensive reviews of constraints on $G$ and newer developments, see [35-37].

It is the nucleosynthesis bounds which we look to update in this work. We do this by (i) using up-to-date measurements of the primordial element abundances reported by the PDG [38], (ii) including a weak determination of the baryon density [17] to alleviate the degeneracy between $\Omega_{\mathrm{b}} h^{2}$ and $G$ in the deuterium abundance, (iii) accurately accounting for incomplete neutrino decoupling following [39,40], and (iv) making use of the state-of-the-art BBN code PRIMAT [41] which has updated nuclear reaction rates and accounts for many corrections to the weak reaction rates. The methodology applied here follows that in [42].

\section{Cosmological implications}

We can understand the effect on the primordial abundances of a different value of $G$ during nucleosynthesis, $G_{\mathrm{BBN}} \neq G_{0}$, in terms of the Hubble expansion rate $H \propto \sqrt{G}$. The dominant effect of an increased (decreased) expansion rate is to
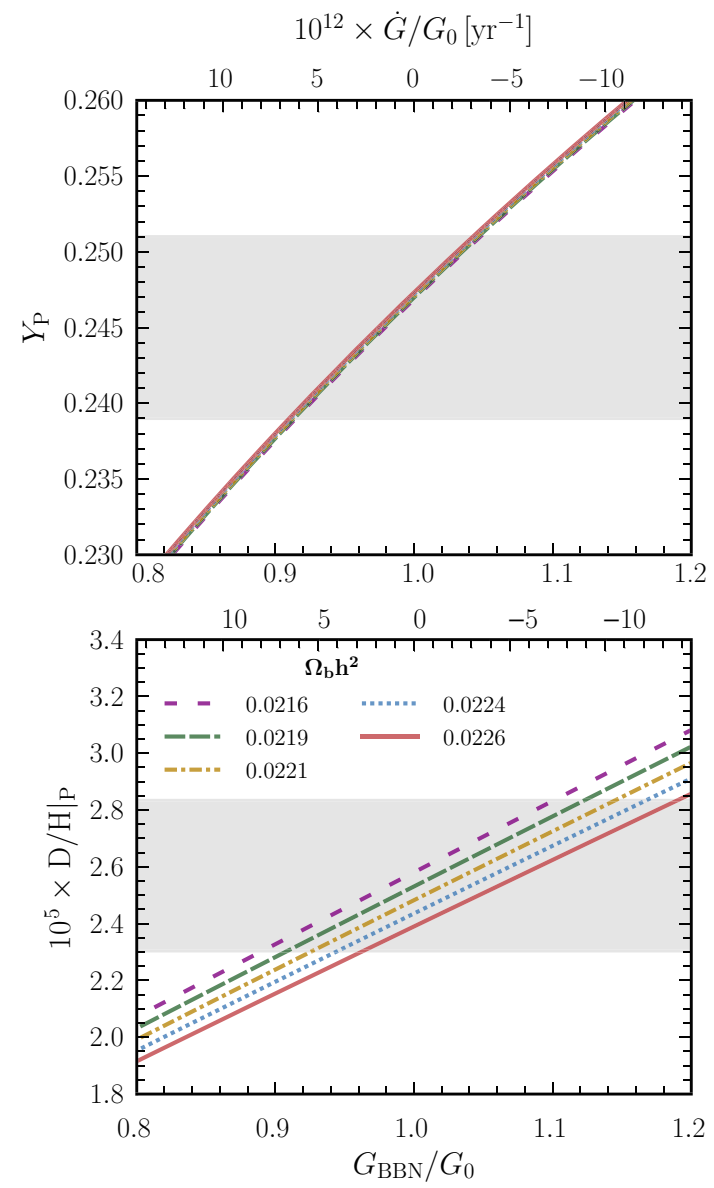

Fig. 1 The variation in the primordial helium abundance (upper) and the primordial deuterium abundance (lower) as a function of $G_{\mathrm{BBN}} / G_{0}$. The grey bands correspond to the $2 \sigma$ confidence intervals from the astrophysical measurements in Eqs. (2) and (3). The different lines correspond to representative values of the baryon density $\Omega_{\mathrm{b}} h^{2}$

alter the time at which various weak and nuclear processes freeze-out. In particular, the proton-to-neutron conversion processes and the $p+n \leftrightarrow D+\gamma$ reaction will freezeout earlier (later). This leads to an over(under)-production of both helium and deuterium compared to the case where $G_{\mathrm{BBN}}=G_{0}$. This is seen clearly in Fig. 1 . From just these figures and the errors in the measurements of the abundances (indicated by the grey bands), we expect that our analysis will be able to constrain variations in $G$ below the $10 \%$ level.

\section{Methodology}

To derive the bounds on the variation of the gravitational constant, we follow the approach presented in [42]. We make use of the publicly available code NUDEC_BSM $[39,40]$ to compute the background cosmology, including the effects of non-instantaneous neutrino decoupling. The relevant cosmological parameters are subsequently forwarded to the state- 
of-the-art BBN code PRIMAT [41], which takes care of the nuclear reaction network and time evolution of primordial abundances. In all simulations we take the neutron lifetime to be the default value in PRIMAT: $\tau_{n}=879.5 \mathrm{~s}$. The abundances scale with $G / G_{0}$ in a way that closely matches the semi-analytic relations given in [43].

To quantify the effect of a time variation in $G$ we choose to parametrise the evolution as a slowly evolving linear function of time $t$ [35],

$G(t)=G_{\mathrm{BBN}}+\dot{G} \times\left(t-t_{\mathrm{BBN}}\right)$,

so that $G_{0}=G_{\mathrm{BBN}}+\dot{G}\left(t_{0}-t_{\mathrm{BBN}}\right)$. Here $t_{0}$ is the current age of the Universe such that $\left(t_{0}-t_{\mathrm{BBN}}\right) \simeq 13.8 \mathrm{Gyr}$ [44]. This slowly varying function ensures that the assumption of a constant $G$ during the cosmologically very short period of primordial nucleosynthesis is an excellent approximation. In the Sect. 5, we will quote bounds on both $G_{\mathrm{BBN}} / G_{0}$ and $\dot{G} / G_{0}$, where the latter can be derived from Eq. (1).

\section{Data analysis}

We make use of the observed primordial abundances of helium and deuterium as reported by the PDG [38]. At 68\% CL, these are:

$Y_{\mathrm{P}}=0.245 \pm 0.003$,

$\mathrm{D} /\left.\mathrm{H}\right|_{\mathrm{P}}=(2.569 \pm 0.027) \times 10^{-5}$.

We also include theoretical errors in the predictions of $Y_{\mathrm{P}}$ and $\mathrm{D} /\left.\mathrm{H}\right|_{\mathrm{P}}$ due to uncertainties in the various nuclear reaction rates and the neutron lifetime [43];

$$
\begin{aligned}
& \sigma\left(Y_{\mathrm{P}}\right)^{\text {Theo }}=0.00018, \\
& \sigma\left(\mathrm{D} /\left.\mathrm{H}\right|_{\mathrm{P}}\right)^{\text {Theo }}=0.13 \times 10^{-5} .
\end{aligned}
$$

To quantify deviations from the measured primordial abundances due to changes in the gravitational constant, we construct a $\chi^{2}$ for BBN as follows:

$$
\begin{aligned}
\chi_{\mathrm{BBN}}^{2}= & \frac{\left[Y_{\mathrm{P}}-Y_{\mathrm{P}}^{\mathrm{Obs}}\right]^{2}}{\sigma^{2}\left(Y_{\mathrm{P}}\right)^{\text {Theo }}+\sigma^{2}\left(Y_{\mathrm{P}}\right)^{\mathrm{Obs}}} \\
& +\frac{\left[\mathrm{D} /\left.\mathrm{H}\right|_{\mathrm{P}}-\mathrm{D} /\left.\mathrm{H}\right|_{\mathrm{P}} ^{\mathrm{Obs}}\right]^{2}}{\sigma^{2}\left(\mathrm{D} /\left.\mathrm{H}\right|_{\mathrm{P}}\right)^{\text {Theo }}+\sigma^{2}\left(\mathrm{D} /\left.\mathrm{H}\right|_{\mathrm{P}}\right)^{\mathrm{Obs}}} .
\end{aligned}
$$

We are also interested in including a conservative determination of the baryon density to lift the degeneracy between $\Omega_{\mathrm{b}} h^{2}$ and $G$. Using directly the posterior values from the baseline $\Lambda$ CDM Planck 2018 analysis will not be satisfactory, because $G$ is kept constant there. Instead, we use the results of [17], who carry out a Planck likelihood analysis including variations in $G_{\mathrm{CMB}}$. In Table I of [17], they find that the mean baryon density exactly matches that of the base Planck 2018 TTTEEE + lowE analysis within ACDM [44], albeit with twice as large error bars:

$\left.\Omega_{\mathrm{b}} h^{2}\right|^{\mathrm{Obs}}=0.02236, \quad \sigma\left(\Omega_{\mathrm{b}} h^{2}\right)=0.00030$.

This allows us to define an extended $\chi^{2}$ for $\mathrm{BBN}+\Omega_{\mathrm{b}} h^{2}$,

$\chi_{\mathrm{BBN}+\Omega_{\mathrm{b}} h^{2}}^{2}=\chi_{\mathrm{BBN}}^{2}+\frac{\left[\Omega_{\mathrm{b}} h^{2}-\left.\Omega_{\mathrm{b}} h^{2}\right|^{\mathrm{Obs}}\right]^{2}}{\sigma^{2}\left(\Omega_{\mathrm{b}} h^{2}\right)}$.

For both the pure BBN analysis and the extended scenario, we compute the relevant $\chi^{2}$ on a grid of $\left(\Omega_{\mathrm{b}} h^{2}, G_{\mathrm{BBN}} / G_{0}\right)$. We then marginalize over the baryon density to find a 1-D $\chi^{2}\left(G_{\mathrm{BBN}} / G_{0}\right)$. To rule out values of $G_{\mathrm{BBN}} / G_{0}$, we compare the computed statistics to critical values of the 1-D $\chi^{2}$ distribution. In particular, at $2 \sigma$, we rule out a scenario if $\Delta \chi^{2} \equiv \chi^{2}-\chi_{\min }^{2} \geq 4$.

\section{Results}

We show the cosmological implications of a variation in the gravitational constant on the primordial helium and deuterium abundances in Fig. 1. It is evident that measurements of both primordial abundances are able to strongly constrain a deviation of $G$ from today's value. Moreover, we see that the impact of a higher value of $G_{\mathrm{BBN}} / G_{0}$ can be compensated by a higher value of the baryon density $\Omega_{\mathrm{b}} h^{2}$, which introduces a degeneracy in the $\left(\Omega_{\mathrm{b}} h^{2}, G_{\mathrm{BBN}} / G_{0}\right)$ plane. This is because changes in the primordial deuterium abundance are linearly proportional to changes in the baryon density $\left(\Delta \mathrm{D} /\left.\mathrm{H}\right|_{\mathrm{P}} \propto-\Delta \Omega_{\mathrm{b}} h^{2}\right)$, while the primordial helium abundance is only logarithmically dependent on $\Omega_{\mathrm{b}} h^{2}$ [41].

In Fig. 2 we show the $1 \sigma$ and $2 \sigma$ confidence intervals in the $\left(\Omega_{\mathrm{b}} h^{2}, G_{\mathrm{BBN}} / G_{0}\right)$ plane and $\Delta \chi^{2}$ as a function of the variation in $G$ for the two benchmark analyses considered here. At $95.4 \%$ CL, we obtain:

$\begin{array}{ll}G_{\mathrm{BBN}} / G_{0}=0.98_{-0.06}^{+0.06} & \mathrm{BBN}, \\ G_{\mathrm{BBN}} / G_{0}=0.99_{-0.05}^{+0.06} & \mathrm{BBN}+\Omega_{\mathrm{b}} h^{2} .\end{array}$

Alternatively, assuming a linear time evolution of the gravitational constant as described in Eq. (1), these bounds can be translated into a constraint on $\dot{G} / G_{0}$, which at $95.4 \% \mathrm{CL}$ reads:

$$
\begin{aligned}
& \frac{\dot{G}}{G_{0}}=1.4_{-4.7}^{+4.4} \times 10^{-12} \text { year }^{-1} \text { BBN }, \\
& \frac{\dot{G}}{G_{0}}=0.7_{-4.3}^{+3.8} \times 10^{-12} \text { year }^{-1} \mathrm{BBN}+\Omega_{\mathrm{b}} h^{2} .
\end{aligned}
$$



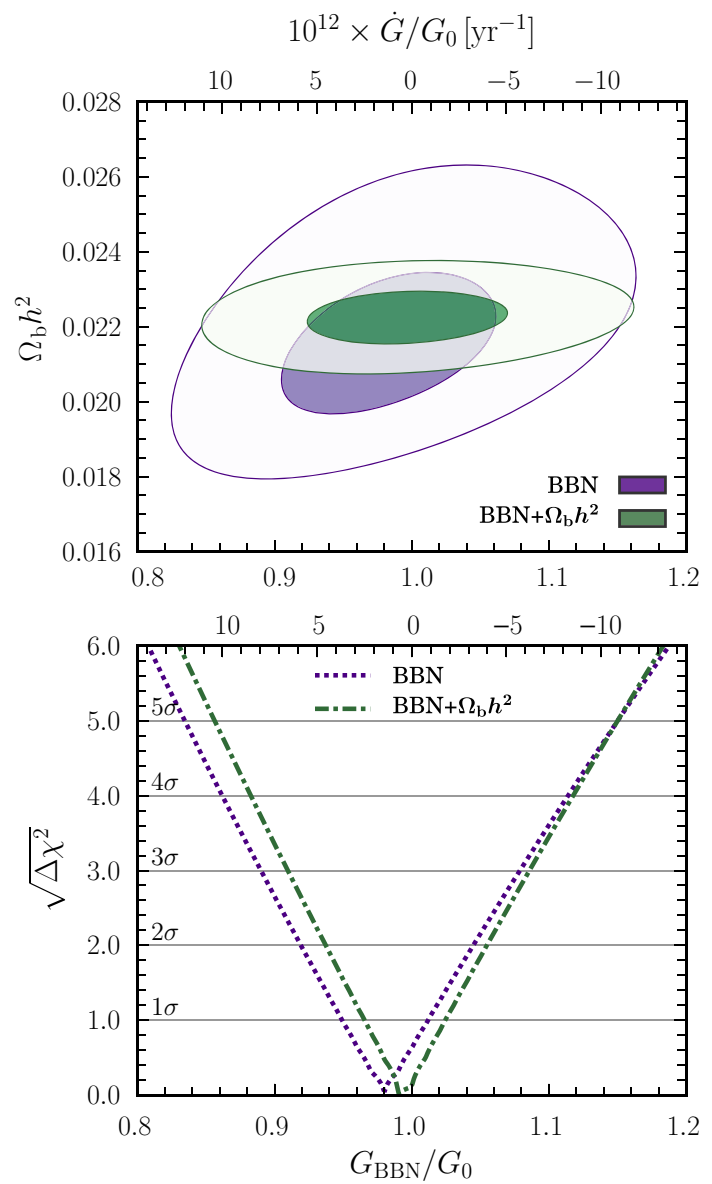

Fig. 2 Contour plot showing the $1 \sigma$ and $2 \sigma$ confidence intervals in the $\left(\Omega_{\mathrm{b}} h^{2}, G_{\mathrm{BBN}} / G_{0}\right)$ plane (upper) and marginalized $\Delta \chi^{2}$ as a function of $G_{\mathrm{BBN}} / G_{0}$ (lower). The dotted lines correspond to BBN constraints and dash-dotted to BBN $+\Omega_{\mathrm{b}} h^{2}$ bounds

Moreover, the BBN $+\Omega_{\mathrm{b}} h^{2}$ analysis highly disfavours a fractional deviation of $G$ larger than $\sim 10 \%$ (at more than $5 \sigma$ ), while a similar conclusion can be drawn in the BBNonly analysis for deviations larger than $\sim 20 \%$. This difference arises because a restriction on $\Omega_{\mathrm{b}} h^{2}$ lifts the degeneracy with $G$ regarding the primordial deuterium abundance. Note that our constraints improve on the previous $1 \sigma$ primordial nucleosynthesis bounds $[33,34]$ by approximately a factor of 10.

As a final comment, other references, such as [41], quote an error on the deuterium value that is approximately 4 times smaller than the one used here. Running our analysis with this lower error, we find no change in the BBN only bounds while the $\mathrm{BBN}+\Omega_{\mathrm{b}} h^{2}$ constraints strengthen by $\sim 30 \%$.

\section{Conclusions}

Big Bang Nucleosynthesis is sensitive to changes in the value of the gravitational constant in the early Universe. By using current measurements of the primordial abundances of helium and deuterium we have shown that at $95.4 \% \mathrm{CL}$, $G_{\mathrm{BBN}} / G_{0}=0.98_{-0.06}^{+0.06}$ and $G_{\mathrm{BBN}} / G_{0}=0.99_{-0.05}^{+0.06}$ if $\Omega_{\mathrm{b}} h^{2}$ measurements from Planck are also accounted for. Assuming a very slow linear time evolution of $G$, these constraints map into a bound on the time variation at $95.4 \% \mathrm{CL}$ of $\dot{G} / G_{0}=$ $1.4_{-4.7}^{+4.4} \times 10^{-12} \mathrm{yr}^{-1}$ and $\dot{G} / G_{0}=0.7_{-4.3}^{+3.8} \times 10^{-12} \mathrm{yr}^{-1}$ respectively. These constraints are competitive and complementary to those from CMB, stellar, solar system, pulsar timing, gravitational waves and lunar laser ranging measurements.

Acknowledgements We would like to thank A. Notari, G. Ballesteros and F. Rompineve for their very helpful correspondence on the first version of this manuscript. We acknowledge the use of the public cosmological code PRIMAT [41]. ME and MF are supported by the European Research Council under the European Union's Horizon 2020 program (ERC Grant Agreement No 648680 DARKHORIZONS). In addition, the work of MF was supported partly by the STFC Grant ST/P000258/1. JA is a recipient of an STFC quota studentship. NS is a recipient of a King's College London NMS Faculty Studentship.

Data Availability Statement This manuscript has no associated data or the data will not be deposited. [Authors' comment: This article is based on data presented in Ref. [38].]

Open Access This article is licensed under a Creative Commons Attribution 4.0 International License, which permits use, sharing, adaptation, distribution and reproduction in any medium or format, as long as you give appropriate credit to the original author(s) and the source, provide a link to the Creative Commons licence, and indicate if changes were made. The images or other third party material in this article are included in the article's Creative Commons licence, unless indicated otherwise in a credit line to the material. If material is not included in the article's Creative Commons licence and your intended use is not permitted by statutory regulation or exceeds the permitted use, you will need to obtain permission directly from the copyright holder. To view a copy of this licence, visit http://creativecomm ons.org/licenses/by/4.0/.

Funded by $\mathrm{SCOAP}^{3}$.

\section{References}

1. P.A.M. Dirac, The cosmological constants. Nature (London) 139, 323 (1937). https://doi.org/10.1038/139323a0

2. T. Kaluza, Zum Unitätsproblem der Physik. Sitzungsber. Preuss. Akad. Wiss. Berlin (Math. Phys.) 1921, 966 (1921). https://doi. org/10.1142/S0218271818700017. (arXiv:1803.08616)

3. O. Klein, Quantum theory and five-dimensional theory of relativity (in German and English). Z. Phys. 37, 895 (1926). https://doi.org/ 10.1007/BF01397481

4. C. Brans, R.H. Dicke, Mach's principle and a relativistic theory of gravitation. Phys. Rev. 124, 925 (1961). https://doi.org/10.1103/ PhysRev.124.925

5. M.R. Douglas, S. Kachru, Flux compactification. Rev. Mod. Phys. 79, 733 (2007). https://doi.org/10.1103/RevModPhys.79. 733. (arXiv:hep-th/0610102) 
6. B. Bertotti, L. Iess, P. Tortora, A test of general relativity using radio links with the Cassini spacecraft. Nature 425, 374 (2003). https://doi.org/10.1038/nature01997

7. J. Khoury, A. Weltman, Chameleon fields: awaiting surprises for tests of gravity in space, Phys. Rev. Lett. 93, 171104 (2004). https://doi.org/10.1103/PhysRevLett.93.171104. (arXiv: astro-ph/0309300)

8. G.W. Biedermann, X. Wu, L. Deslauriers, S. Roy, C. Mahadeswaraswamy, M.A. Kasevich, Testing gravity with coldatom interferometers. Phys. Rev. A91, 033629 (2015). https://doi. org/10.1103/PhysRevA.91.033629. (arXiv:1412.3210)

9. M.J. Duff, How fundamental are fundamental constants? Contemp. Phys. 56, 35 (2015). https://doi.org/10.1080/00107514. 2014.980093. (arXiv: 1412.2040)

10. M. Peimbert, A. Peimbert, V. Luridiana, A new determination of the primordial helium abundance, in Revista Mexicana de Astronomia y Astrofisica Conference Series, vol. 49 of Revista Mexicana de Astronomia y Astrofisica, vol. 27, pp. 181-181 (2017)

11. E. Aver, K.A. Olive, E.D. Skillman, The effects of He I ?10830 on helium abundance determinations. JCAP 1507, 011 (2015). https:// doi.org/10.1088/1475-7516/2015/07/011. (arXiv: 1503.08146)

12. Y.I. Izotov, T.X. Thuan, N.G. Guseva, A new determination of the primordial $\mathrm{He}$ abundance using the $\mathrm{He}$ i $\lambda 10830 \AA$ emission line: cosmological implications. Mon. Not. R. Astron. Soc. 445, 778 (2014). https://doi.org/10.1093/mnras/stu1771. (arXiv:1408.6953)

13. R.J. Cooke, M. Pettini, K.M. Nollett, R. Jorgenson, The primordial deuterium abundance of the most metal-poor damped Ly $\alpha$ system. Astrophys. J. 830, 148 (2016). https://doi.org/10.3847/ 0004-637X/830/2/148. (arXiv:1607.03900)

14. S.A. Balashev, E.O. Zavarygin, A.V. Ivanchik, K.N. Telikova, D.A. Varshalovich, The primordial deuterium abundance: subDLA system at $z_{\mathrm{abs}}=2.437$ towards the QSO J $1444+2919$, Mon. Not. R. Astron. Soc. 458, 2188 (2016). https://doi.org/10.1093/mnras/ stw356. (arXiv:1511.01797)

15. E.O. Zavarygin, J.K. Webb, V. Dumont, S. Riemer-Sørensen, The primordial deuterium abundance at $\mathrm{z}_{a b s}=2.504$ from a high signal-to-noise spectrum of Q1009+2956. Mon. Not. R. Astron. Soc. 477, 5536 (2018). https://doi.org/10.1093/mnras/sty1003. (arXiv:1706.09512)

16. S. Riemer-Sørensen, S. Kotuš, J.K. Webb, K. Ali, V. Dumont, M.T. Murphy et al., A precise deuterium abundance: remeasurement of the $\mathrm{z}=3.572$ absorption system towards the quasar PKS1937-101. Mon. Not. R. Astron. Soc. 468, 3239 (2017). https://doi.org/10. 1093/mnras/stx681. (arXiv:1703.06656)

17. Y. Bai, J. Salvado, B.A. Stefanek, Cosmological constraints on the gravitational interactions of matter and dark matter, JCAP 1510, 029 (2015). https://doi.org/10.1088/1475-7516/2015/10/ 029. (arXiv: 1505.04789)

18. J. Ooba, K. Ichiki, T. Chiba, N. Sugiyama, Cosmological constraints on scalar-tensor gravity and the variation of the gravitational constant. PTEP 2017, $043 E 03$ (2017). https://doi.org/10. 1093/ptep/ptx046. (arXiv: 1702.00742)

19. E. Garcia-Berro, P. Loren-Aguilar, S. Torres, L.G. Althaus, J. Isern, An upper limit to the secular variation of the gravitational constant from white dwarf stars, JCAP 1105, 021 (2011). https://doi.org/ 10.1088/1475-7516/2011/05/021. (arXiv: 1105.1992)

20. J. Mould, S. Uddin, Constraining a possible variation of $G$ with Type Ia supernovae. Publ. Astron. Soc. Aust. 31, 15 (2014). https:// doi.org/10.1017/pasa.2014.9. (arXiv:1402.1534)

21. E.P. Bellinger, J. Christensen-Dalsgaard, Asteroseismic constraints on the cosmic-time variation of the gravitational constant from an ancient main-sequence star. (arXiv:1909.06378)

22. J. Chandler, R. Reasenberg, I. Shapiro, New bounds on G. Bull. Am. Astron. Soc. 25, 1233 (1993)
23. E.V. Pitjeva, N.P. Pitjev, Relativistic effects and dark matter in the Solar system from observations of planets and spacecraft. Mon. Not. R. Astron. Soc. 432, 3431 (2013). https://doi.org/10.1093/ mnras/stt695. (arXiv:1306.3043)

24. A. Fienga, J. Laskar, P. Exertier, H. Manche, M. Gastineau, Tests of general relativity with planetary orbits and Monte Carlo simulations. (arXiv:1409.4932)

25. V.M. Kaspi, J.H. Taylor, M.F. Ryba, High-precision timing of millisecond pulsars. 3: Long-term monitoring of PSRs B1855+09 and B1937+21. Astrophys. J. 428, 713 (1994). https://doi.org/10.1086/ 174280

26. W.W. Zhu et al., Tests of gravitational symmetries with pulsar binary J1713+0747. Mon. Not. R. Astron. Soc. 482, 3249 (2019). https://doi.org/10.1093/mnras/sty2905. (arXiv:1802.09206)

27. J.G. Williams, S.G. Turyshev, D.H. Boggs, Progress in lunar laser ranging tests of relativistic gravity. Phys. Rev. Lett. 93, 261101 (2004). https://doi.org/10.1103/PhysRevLett.93.261101. (arXiv:gr-qc/0411113)

28. F. Hofmann, J. Müller, Relativistic tests with lunar laser ranging. Class. Quant. Grav. 35, 035015 (2018). https://doi.org/10.1088/ 1361-6382/aa8f7a

29. N. Yunes, F. Pretorius, D. Spergel, Constraining the evolutionary history of Newton's constant with gravitational wave observations, Phys. Rev. D81, 064018 (2010). https://doi.org/10.1103/ PhysRevD.81.064018. (arXiv:0912.2724)

30. W. Zhao, B.S. Wright, B. Li, Constraining the time variation of Newton's constant $G$ with gravitational-wave standard sirens and supernovae, JCAP 1810, 052 (2018). https://doi.org/10.1088/ 1475-7516/2018/10/052. (arXiv: 1804.03066)

31. B.A. Campbell, K.A. Olive, Nucleosynthesis and the time dependence of fundamental couplings. Phys. Lett. B345, 429 (1995). https://doi.org/10.1016/0370-2693(94)01652-S. (arXiv:hep-ph/9411272)

32. T. Dent, M. Fairbairn, Time varying coupling strengths, nuclear forces and unification. Nucl. Phys. B653, 256 (2003). https://doi. org/10.1016/S0550-3213(03)00043-9. (arXiv:hep-ph/0112279)

33. C.J. Copi, A.N. Davis, L.M. Krauss, A New nucleosynthesis constraint on the variation of G. Phys. Rev. Lett. 92, 171301 (2004). https://doi.org/10.1103/PhysRevLett.92.171301. (arXiv: astro-ph/0311334)

34. T. Clifton, J.D. Barrow, R.J. Scherrer, Constraints on the variation of $\mathrm{G}$ from primordial nucleosynthesis. Phys. Rev. D71 123526 (2005). https://doi.org/10.1103/PhysRevD.71.123526. (arXiv: astro-ph/0504418)

35. J.-P. Uzan, The fundamental constants and their variation: observational status and theoretical motivations. Rev. Mod. Phys. 75, 403 (2003). https://doi.org/10.1103/RevModPhys.75. 403. (arXiv:hep-ph/0205340)

36. J.-P. Uzan, Varying constants, gravitation and cosmology. Living Rev. Relat. 14, 2 (2011). https://doi.org/10.12942/lrr-2011-2. (arXiv: 1009.5514)

37. C.J.A.P. Martins, The status of varying constants: a review of the physics, searches and implications. (arXiv:1709.02923)

38. ParticleDataGroup collaboration, M. Tanabashi et al., Review of particle physics. Phys. Rev. D98, 030001 (2018). https://doi.org/ 10.1103/PhysRevD.98.030001

39. M. Escudero, Neutrino decoupling beyond the standard model: $\mathrm{CMB}$ constraints on the dark matter mass with a fast and precise $N_{\text {eff }}$ evaluation, JCAP 1902, 007 (2019). https://doi.org/10.1088/ 1475-7516/2019/02/007. (arXiv: 1812.05605)

40. M. Escudero, Precision early universe thermodynamics made simple: $n_{\text {eff }}$ and neutrino decoupling in the standard model and beyond. (arXiv:2001.04466)

41. C. Pitrou, A. Coc, J.-P. Uzan, E. Vangioni, Precision big bang nucleosynthesis with improved Helium-4 predictions. Phys. Rep. 
754, 1 (2018). https://doi.org/10.1016/j.physrep.2018.04.005. (arXiv:1801.08023)

42. N. Sabti, J. Alvey, M. Escudero, M. Fairbairn, D. Blas, Refined bounds on $\mathrm{MeV}$-scale thermal dark sectors from BBN and the CMB. (arXiv:1910.01649)
43. B.D. Fields, K.A. Olive, T.-H. Yeh, C. Young, Big-bang nucleosynthesis after planck. (arXiv:1912.01132)

44. PlanCK collaboration, N. Aghanim et al., Planck 2018 results. VI. Cosmological parameters. (arXiv:1807.06209) 